\title{
Combined logarithmic and exponential function model for fitting postseismic GNSS time series after 2011 Tohoku-Oki earthquake
}

\author{
Mikio Tobita*
}

\begin{abstract}
The time series of a postseismic deformation is commonly fitted by a logarithmic or exponential decay function. However, the high-quality postseismic Global Navigation Satellite System (GNSS) time series of the 2011 Mw 9TohokuOki earthquake indicates that a single decay function cannot be used to represent the postseismic behaviour. We therefore combined the logarithmic (log) and exponential (exp) decay functions and developed methods for obtaining global solutions using nonlinear least squares calculations for such complex functions. Our models significantly improved the fitting performance of the postseismic time series and the prediction performance of the evolution of postseismic deformation. The solutions obtained by the proposed models and methods enabled distinction between the contributions of the log and exp functions, and explanation of characteristic phenomena such as the subsidence that occurs immediately after an earthquake is reversed to an uplift. The analysis of the solutions may suggest that there has been a continuous increase in the contribution of viscoelastic relaxation to postseismic deformation in eastern Japan, whereas the contribution of afterslip has rapidly decreased. The short-term prediction performance and the universal applicability of the proposed models to the Tohoku-Oki earthquake have contributed to the detection of a slow-slip event in the Tokai region. Rather than the existence of a unique single relaxation time for each surface site, our results suggest a unique single relaxation time for each postseismic deformation mechanism at a given subsurface location. Although the predictions were highly dependent on the assigned steady velocities and the long-term relaxation time constants, they indicate that the coseismic subsidence of the Yamoto station in Miyagi prefecture will recover around the year 2020. The estimated relaxation time constants of the present models appeared to be uniform throughout eastern Japan.
\end{abstract}

Keywords: 2011 Tohoku-Oki earthquake, Postseismic deformation, Function fitting, Viscoelastic relaxation, Afterslip, GNSS, Universal relaxation time

\section{Background}

Since the 2011 off the Pacific coast of Tohoku Earthquake (Tohoku-Oki earthquake), crustal deformation has continued to occur in eastern Japan (Ozawa et al. 2012; GSI 2015a). The primary mechanisms of the postseismic deformations are commonly recognized as afterslip and viscoelastic relaxations. However, the relative contributions of the afterslip and viscoelastic relaxations remain unclear (Sun and Wang 2015). An understanding of the

*Correspondence: tobita-m96mh@mlit.go.jp Geospatial Information Authority of Japan, Kitasato-1, Tsukuba, Ibaraki 305-0811, Japan relevant mechanisms will contribute to the assessment of earthquake potential in the Japan Trench region and neighbouring regions along the Nankai Trough. For example, the postseismic deformation caused by the Tohoku-Oki earthquake is still being observed in 2015, including in the Tokai region, which is located on the eastern part of the Nankai Trough. The deformation has been a noise source in the monitoring of aseismic slips that might be related to the expected Tokai earthquake. The monitoring is part of the monthly assessments of the Earthquake Assessment Committee (EAC) of Japan, and the elimination of the noise is urgently required.

\section{Springer}

(c) 2016 Tobita. This article is distributed under the terms of the Creative Commons Attribution 4.0 International License (http:// creativecommons.org/licenses/by/4.0/, which permits unrestricted use, distribution, and reproduction in any medium, provided you give appropriate credit to the original author(s) and the source, provide a link to the Creative Commons license, and indicate if changes were made. 
Attempts have been made to use function fitting regression analysis to investigate postseismic deformation (Takahashi et al. 2004; Hashimoto et al. 2006; Nishimura 2014). The time series of a postseismic deformation is commonly fitted by a logarithmic or exponential decay function. However, the postseismic time series of the 2011 Tohoku-Oki earthquake observed by the GNSS Earth Observation Network System (GEONET) indicates that a single decay function cannot be used to represent the postseismic behaviour (GSI 2014; Tobita and Akashi 2015; Fig. 2).

Global Navigation Satellite System (GNSS) time series data with a high signal-to-noise ratio $(\mathrm{S} / \mathrm{N})$ may be used to select an appropriate function fitting model. Hence, in the present study, we derived a fitting function for better explanation of the GNSS time series and prediction of the short-term evolution of postseismic deformation. We utilized summation of decay functions and developed methods for obtaining global solutions by nonlinear least squares calculations using complex functions. We evaluated the fitting and prediction performances of the combined function models, as well as their limitations. Moreover, we demonstrated the universality of the determined relaxation time in the Tohoku-Oki earthquake.

\section{Methods}

We used the daily coordinates of F3 solutions (Nakagawa et al. 2009) provided by the GEONET, which consists of $\sim 1300$ permanent GNSS stations in Japan and is operated by the Geospatial Information Authority of Japan (GSI), known before 2010 as the Geographical Survey Institute. Figure 1 shows a location map of the selected 246 GNSS stations with relatively high-quality data among the GEONET stations in eastern Japan that were considered in this study. The GNSS time series data observed after the 2011 Tohoku-Oki earthquake were mainly analysed. The Fukue GNSS station (station ID: 950462) in Nagasaki prefecture was used as the reference station in this study.

The original GNSS time series contained offsets caused by earthquakes, and the offsets hampered the derivation of the fitting functions. We therefore removed those caused by the M 6.0 23 March 2011 Fukushimaken-Hamadori earthquake, M 7.17 April 2011 Miyagiken-Oki earthquake, M 7.0 11 April 2011 Fukushimaken-Hamadori earthquake, M 6.4 12 April 2011 Chibaken-Toho-Oki earthquake, M 6.923 June 2011 Iwateken-Oki earthquake, M 5.4 29 September 2011 Fukushimaken-Hamadori earthquake, M 6.1 14 March 2012 Chibaken-Toho-Oki earthquake, M 6.9 17 February 2015 Sanriku-Oki earthquake, and M 6.8 13 May 2015 Miyagiken-Oki earthquake.

The Yamoto, Choshi, Miyako, and Minase stations [(a)-(d) in Fig. 1] were used for the main modelling for

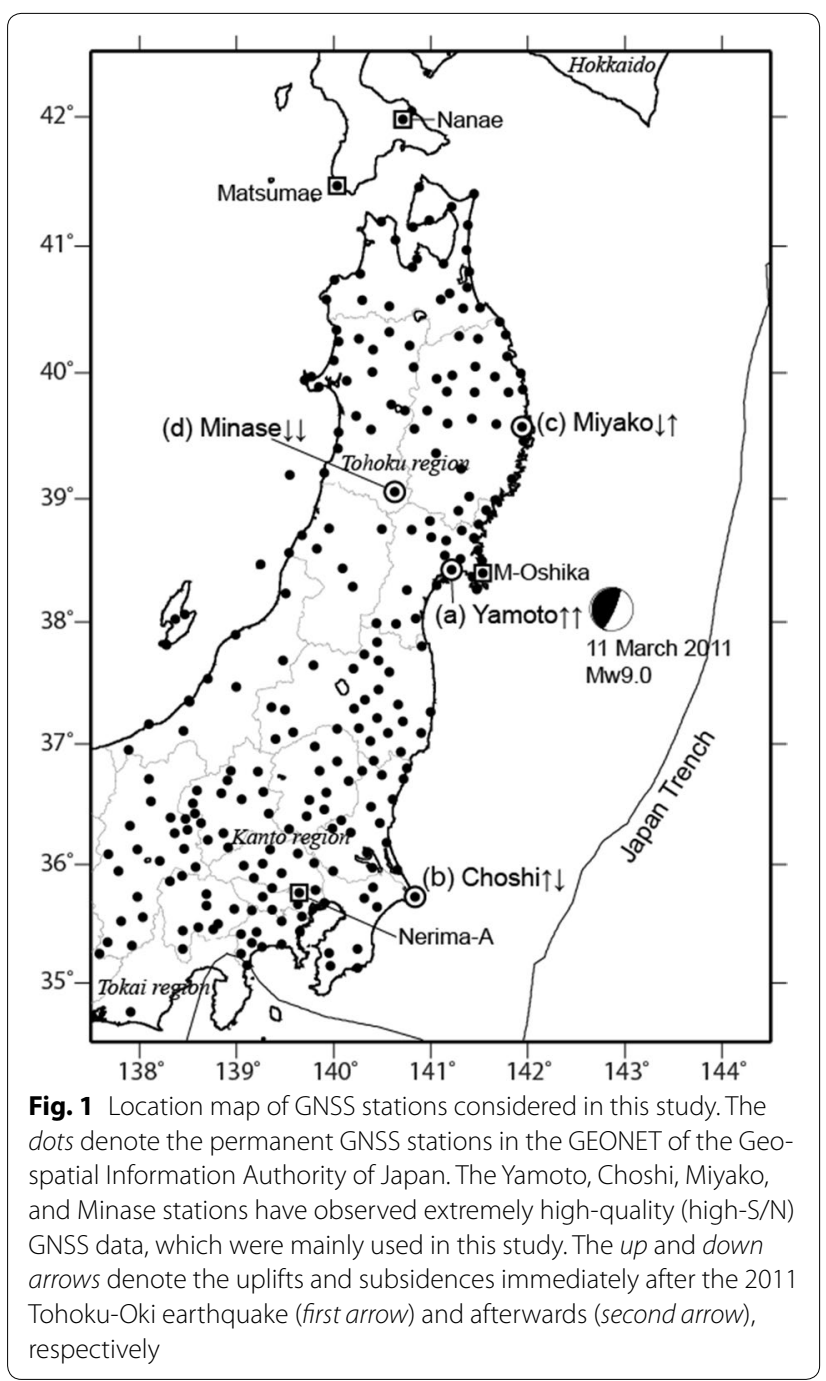

two reasons. Firstly, among all the GEONET stations, these four stations have acquired extremely high-quality GNSS data from the viewpoint of low noise and seasonal variations. Secondly, although the four stations had all coseismically subsided at the time of the 2011 TohokuOki earthquake, the progresses of their vertical postseismic deformations have been different, as represented by the black and grey observed positions in Fig. 3. The Yamoto station (a) in Miyagi prefecture uplifted rapidly after the earthquake, and the uplift has continued at a rate of $\sim 5 \mathrm{~cm} / \mathrm{yr}$ since 2013 . The Choshi station (b) in Chiba prefecture uplifted rapidly immediately after the earthquake, but the vertical movement slowed down and slightly subsided in 2015. The Miyako station (c) in Iwate prefecture subsided rapidly after the earthquake, but this was reversed to a slow uplift around 2012. The Minase station (d) in Akita prefecture experienced rapid subsidence after the earthquake, and the subsidence was still 
observed in 2015. These four stations exhibit representative regional crustal deformations and thus provided strong constraints for our nonlinear least squares calculations, as described below.

Although we investigated the use of a natural logarithmic function (log), natural exponential function (exp), double natural exponential functions with short and long relaxation time constants (exp $+\exp$ ), and double natural logarithmic functions with short and long relaxation time constants $(\log +\log )$ as the fitting model functions for the GNSS time series data, this paper mainly discusses the use of the following three model functions, which were found to be better than the above four:

$$
\begin{aligned}
D(t)= & a \ln (1+t / b)+c-d \exp (-t / e)+V t, \\
D(t)= & a \ln (1+t / b)+c+d \ln (1+t / e) \\
& -f \exp (-t / g)+V t, \\
D(t)= & a \ln (1+t / b)+c-d \exp (-t / e) \\
& -f \exp (-t / g)+V t,
\end{aligned}
$$

where $D(t)$ denotes a displacement component (east, north, or up); $t$ is the time in days relative to the occurrence of the main shock (11 March 2011); $b, e$, and $g$ denote the timescale parameters of the logarithmic or exponential decays (relaxation times); and $V$ is the steady velocity. Here, we refer to the model represented by Eq. (1) as Model 1 or $\log +$ exp model, that represented by Eq. (2) as Model 2 or $\log +\log +\exp$ model, and that represented by Eq. (3) as Model 3 or $\log +\exp +\exp$ model. Whereas the parameters $a, b, c, d, e, f$, and $g$ were estimated by the nonlinear least squares method, the steady velocity $V$ at each station was assigned the corresponding value of the interseismic velocity between 1 April 1997 and 31 March 2000. The reason for this and the consequent limitations are discussed below in the subsection titled 'Limitations of Combined log and exp Models' under 'Discussion'.

To evaluate the fitting and prediction performances of these models, we used the Akaike information criteria (AIC) (Akaike 1974). We assigned 731 days (2 years) after the 2011 Tohoku-Oki earthquake as the 'fitting period', and the number of days between the 732nd and 1681th days ( $\sim 2$ years 7 months) as the 'prediction period'. One of the reasons why exact 2.00 years (731 days) was used as the fitting period was to minimize the effect of seasonal noise in the GNSS data on the fitting. The prediction performance was also evaluated by computing the deviation of the latest observed GNSS data from the value predicted (calculated) by the fitted model function.
Generally, it is difficult to obtain a global solution in nonlinear least squares calculations, and obtaining a global solution of a more complex function requires greater effort. We thus addressed the problem using the following procedure. Firstly, we assumed that the relaxation times $b, e$, and $g$ of the three components (east, north, and up, respectively) of the displacements were the same. This was because the parameters represented the characteristic relaxation times of the corresponding source mechanisms (afterslip and viscoelastic relaxation). Secondly, we assumed that the four stations had the same relaxation times $(b, e$, and $g)$. The basis and validity of this assumption are presented under 'Discussion. Thirdly, we used only high-S/N data obtained from the four high-quality stations, which had different vertical movement progressions as noted above. Fourthly, we used multiple starting points, which means that the computations started with different initial values. This adopted approach effectively improved the computability of the global solutions of the nonlinear least squares calculations, as well as the stability of the estimated values of the relaxation time.

\section{Results}

Before presenting the results obtained by the new models, it is necessary to describe those obtained by simple conventional functions. The exponential function (exp), which is the most simple decay function, can only fit the GNSS time series data obtained after the 2011 Tohoku-Oki earthquake for a short period ( $<1$ year), and it generally underestimates the future evolution of the displacements (east component in Fig. 2). While the logarithmic function $(\log )$ is obviously better than the exponential function, it has similar shortcomings. Neither a single log nor single exp function can fit a time series with reversal from subsidence to uplift (up component in Fig. 2). The estimated relaxation time largely depends on the components, i.e. 28 days for an east component and 0.025 days for an up component (Fig. 2), and also significantly depends on the fitting period, i.e. 0.025 days for a 731-day fitting period and $1.3 \times 10^{8}$ days for a 1681-day fitting period. The exp + exp model with its two relaxation time constants is better than both the log and exp models, but is obviously poorer than Models 1,2 , and 3 . Its prediction performance is particularly poorer. Moreover, while the $\log +\log$ model is superior to the exp + exp model, it is not superior to Models 1, 2, and 3 in terms of the fitting and prediction performances.

Figure 3 shows the results of the log + exp model (Model 1) represented by Eq. (1). For the two-year fitting period after the main shock, the fitting performances at all the stations $[(\mathrm{a})-(\mathrm{d})]$ are reasonably good. For 


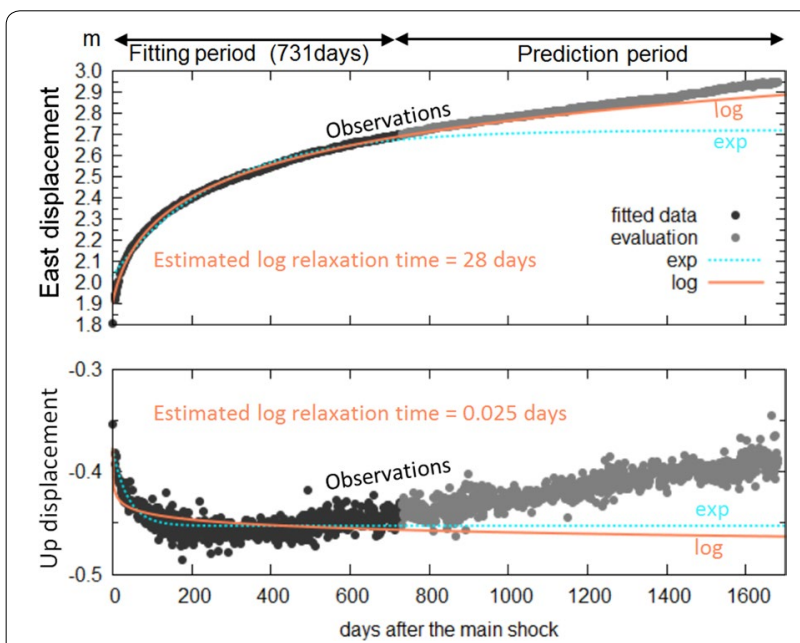

Fig. 2 GNSS time series of Miyako station after the main shock on 11 March 2011, and the time series calculated using the single exp function and the single log function. The black closed circles denote the observed GNSS positions 2 years after the main shock with respect to the position just before the main shock. The exp function (light blue dotted line) and log function (orange line) were, respectively, fitted to the positions observed in the 2-year fitting period between 12 March 2011 and 11 March 2013. These lines were extended to 4.6 years after the main shock based on calculations using each function. The grey closed circles denote the observed GNSS position during the prediction period after the fitting period. Both the fitting and prediction performances of the single exp and single log function are obviously poorer than those of Model 2 in Fig. $4 \mathrm{c}$

example, the vertical displacement of the Miyako station (c) is shown to reverse from subsidence to uplift, which can be explained by a short-term logarithmic subsidence and a relatively longer-term exponential uplift. However, while the east and up components at the Choshi station (b) are well predicted, the predictions for the other stations [(a), (c), and (d)] tend to be underestimated.

Figure 4 shows the results of the $\log +\log +$ exp model (Model 2) represented by Eq. (2). The fitting performances at all the stations [(a)-(d)] are sufficiently good. Moreover, the prediction performance of the model is quite better than that of Model 1. However, a close comparison of the latest observations shown in grey and the calculation results shown in red reveals very small overestimations for the Choshi station (b).

Figure 5 shows the results of the log $+\exp +$ exp model (Model 3) represented by Eq. (3). The fitting performances at all the stations [(a)-(d)] are sufficiently good. The prediction performance is also quite better than that of Model 1. However, a close comparison of the latest observations shown in grey and the calculation results shown in red reveals very small overestimations for the Choshi station (b) and small underestimations of the subsidence of the Minase station (d).
Table 1 summarizes the fitting results of Models 1-3 for the fitting period between 12 March 2011 and 11 March 2013. The AIC values in the table indicate that the fitting performances of Models 2 and 3 are clearly better than that of Model 1, and that Model 3 has a slightly better fitting performance than Model 2. To quantitatively evaluate the prediction performances, the AIC value for each model was calculated using the data for the entire period, i.e. 12 March 2011 to 17 October 2015, which includes the prediction period. The results for Models 1, 2 , and 3 were $-173945,-197678$, and -196954 , respectively. As can be seen, Model 2 has the best prediction performance, although the difference in the prediction performances of Models 2 and 3 is small.

Here, we attempt to predict future postseismic displacements using Model 2 (Fig. 4). The 50-cm coseismic subsidence of the Yamoto station (a) is predicted to recover around the year 2020. Although the Choshi station (b) was rapidly uplifted immediately after the main shock, the vertical movement is predicted to gradually reverse to subsidence. Furthermore, whereas the subsidence of the Miyako station (c) immediately after the main shock has been reversed to an uplift, the uplift is predicted to slow down. The subsidence of the Minase station (d) is predicted to continue, although it is expected to slow down. The contribution of the exponential term possibly viscoelastic relaxation (see "Relationship between postseismic deformation mechanisms and decay functions' under 'Discussion' section) has gradually increased, while the relative contribution of the logarithmic term possibly mainly afterslip (see 'Relationship between postseismic deformation mechanisms and decay functions' under 'Discussion' section) has rapidly decreased.

We examined the universality of our model using the estimated relaxation time constants (Table 1). The relaxation time constants estimated by Model 2 were substituted in Eq. (2), giving

$$
\begin{aligned}
D(t)= & a \ln (1+t / 1.4)+c+d \ln (1+t / 82.0) \\
& -f \exp (-t / 3217.0)+V t .
\end{aligned}
$$

Equation (4) was fitted to the GNSS time series of M-Oshika (059071) in Miyagi prefecture, Nerima-A (081171) in Tokyo, and Matumae (94023) and Nanae (960529) in Hokkaido (Fig. 1). The region in which M-Oshika is located is nearest to the main shock, and its data have the highest $\mathrm{S} / \mathrm{N}$ among the postseismic GNSS data. Nerima-A is far from the source region, and its $\mathrm{S} / \mathrm{N}$ is relatively low. The fitting performance of Eq. (4) was observed to be almost perfect, and its short-term prediction performance was also quite high (Fig. 6a, b). The evolutions of the postseismic vertical displacements of the Matsumae and Nanae stations in Southern Hokkaido appeared to be different. While the Matsumae station 


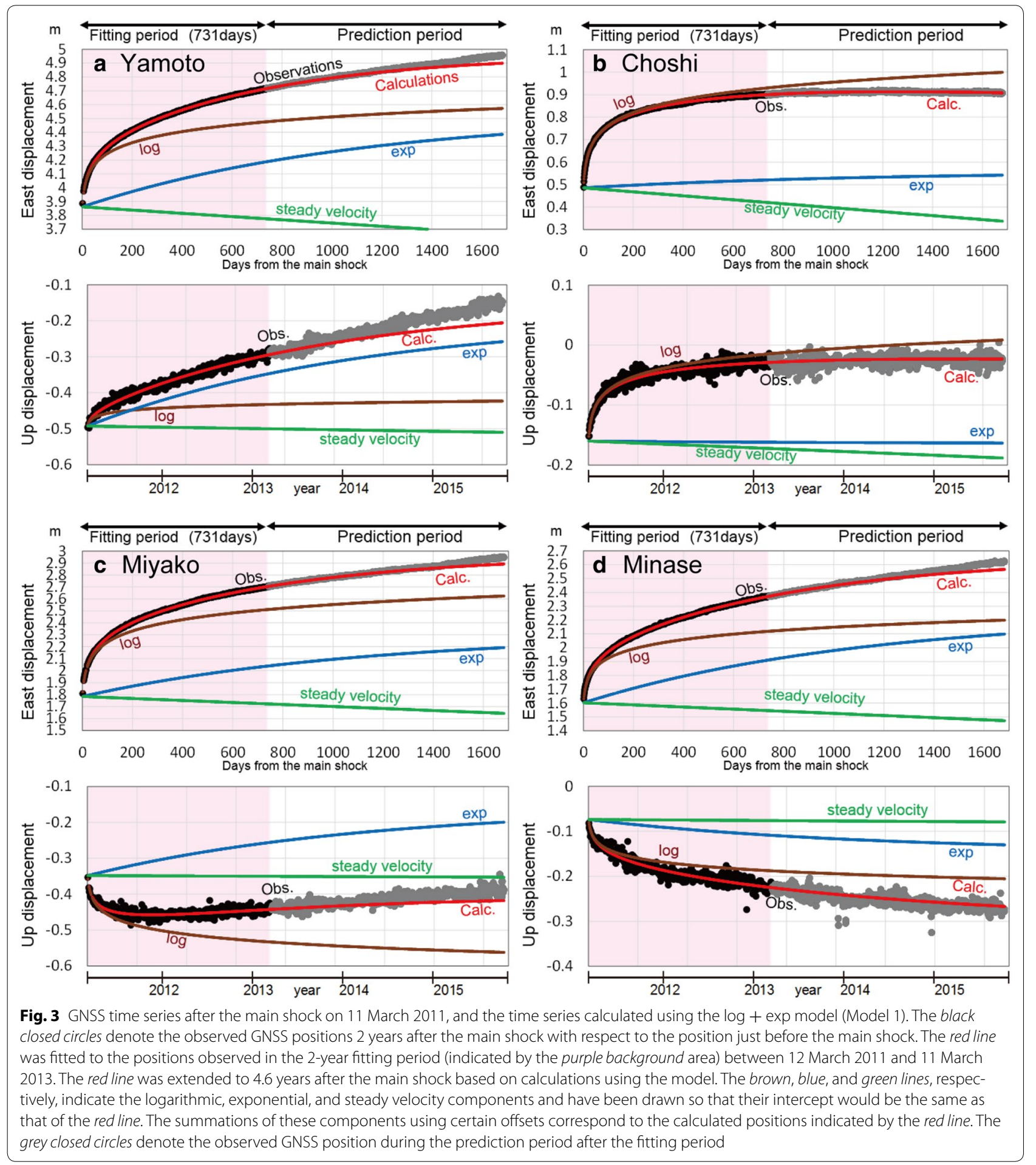

gradually uplifted after the main shock, the Nanae station uplifted rapidly, and this was followed by a rather linear uplift. Although it appeared very difficult to fit such diverse evolution data using fixed relaxation time constants, the results in Fig. 6c, d indicate the satisfactory fitting and short-term prediction performance of Eq. (4). We thus confirmed the universal applicability of the model with the estimated relaxation time constants to the Tohoku-Oki earthquake, at least for the four examined stations. 


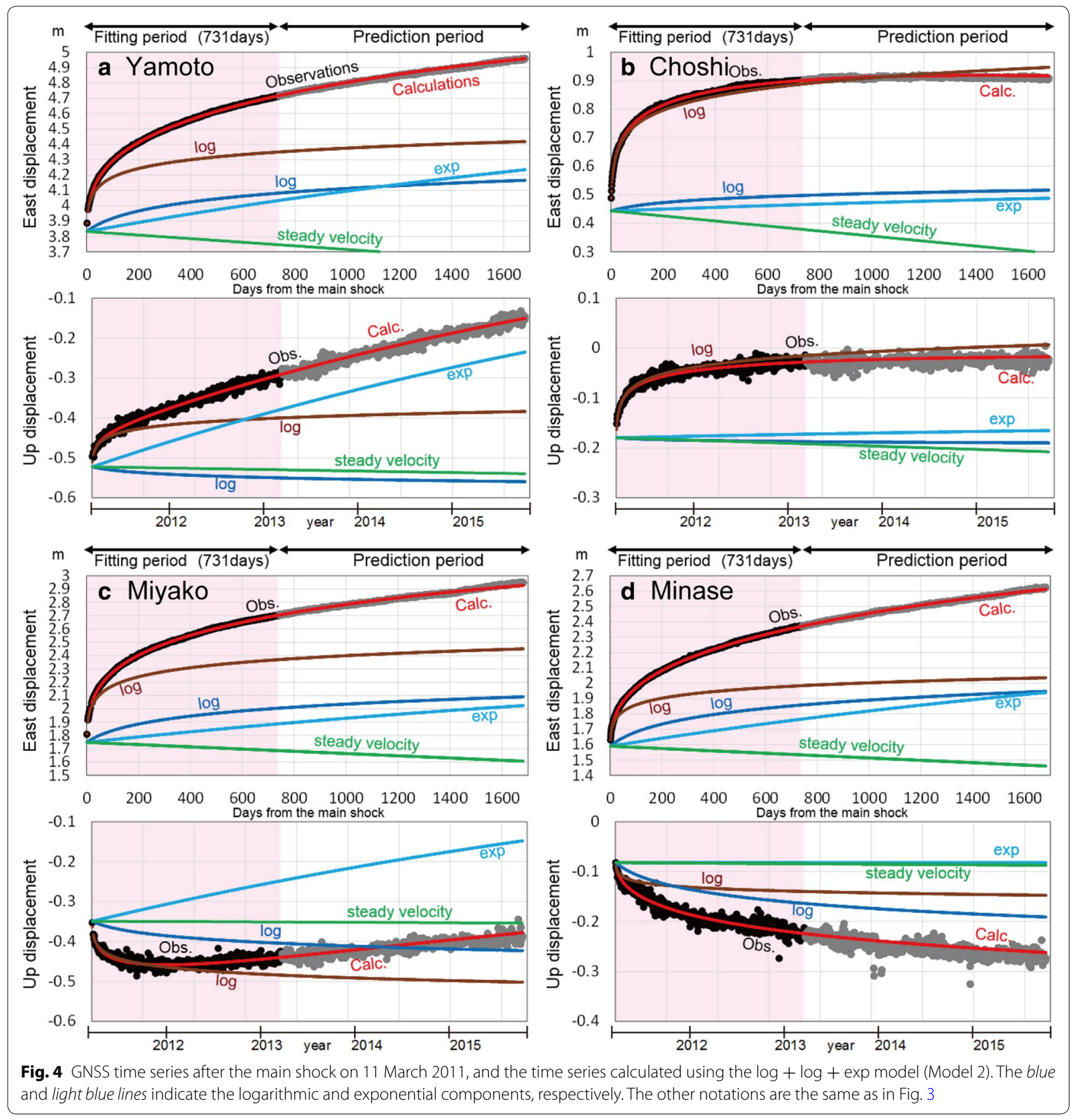

\section{Discussion}

As observed above, we found that a combination of the relaxation times estimated by the simultaneous estimation of the three components of the four stations could be universally applied to GNSS stations in the region. Our results do not suggest a unique single relaxation time for each surface site, but that each postseismic deformation mechanism at a given subsurface location has a unique single relaxation time. Because the fitting and prediction performances of our combined logarithmic and exponential model were reasonably high, we confirmed that each station had observed a surface displacement that is a superposition of the contributions caused by multiple postseismic deformation mechanisms with different timescales, with differing contribution ratios of each mechanism at the different stations. We observed that multiple postseismic deformation mechanisms with different timescales had been continuously active in eastern Japan. 


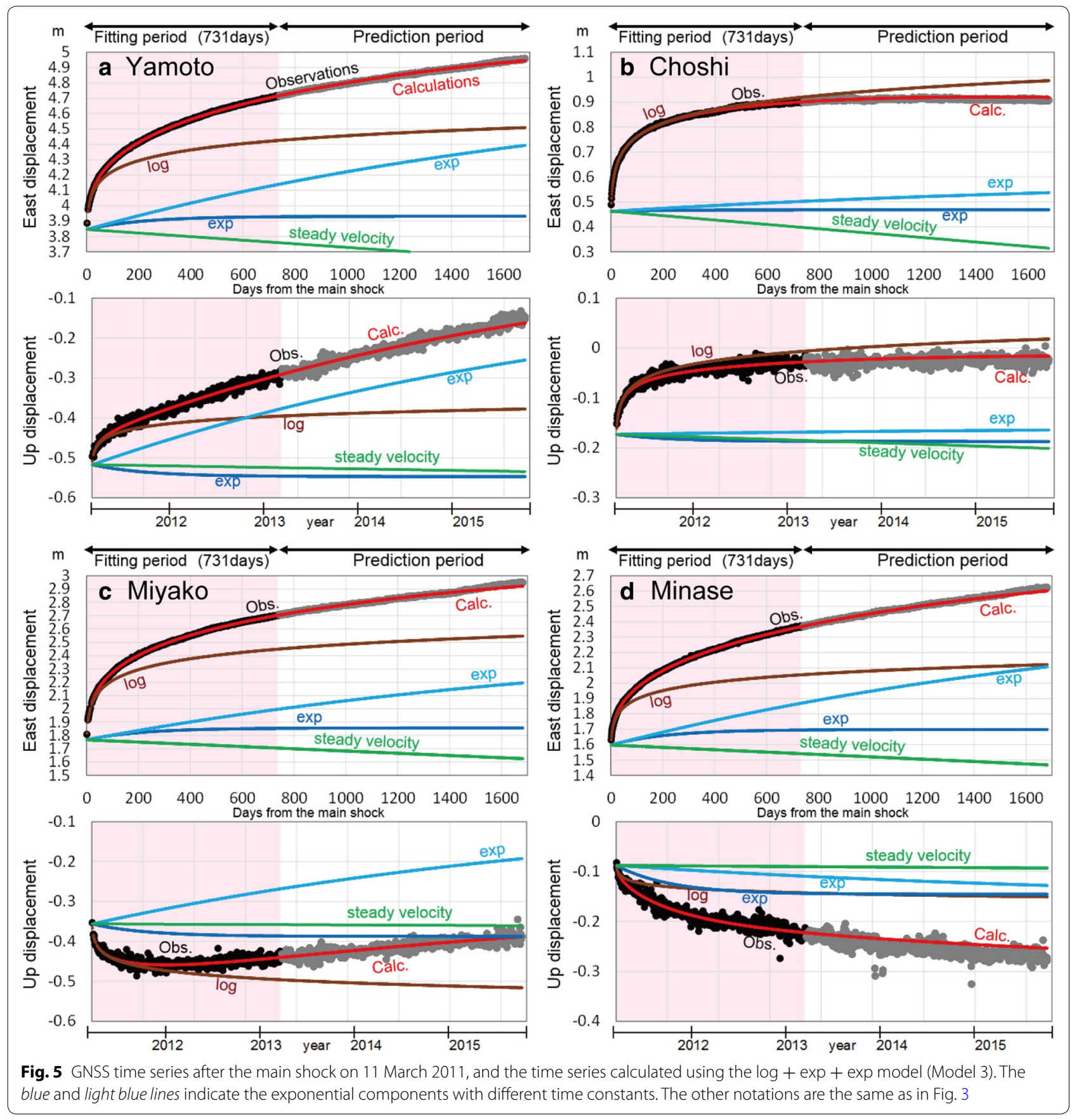

Table 1 Results of fitting to data for two years using Models 1,2 , and 3

\begin{tabular}{llllllll}
\hline $\begin{array}{l}\text { Model } \\
\text { number }\end{array}$ & $\begin{array}{l}\text { Standard } \\
\text { deviation } \\
(\mathbf{m m})\end{array}$ & AIC & \multicolumn{4}{c}{ Relaxation time (days) } \\
\cline { 4 - 7 } & & & Log1 & Log2 & Exp1 & Exp2 \\
\hline 1 & 4.86 & -93387 & 3.9 & N/A & 1110.0 & N/A \\
2 & 4.72 & -93865 & 1.4 & 82.0 & N/A & 3217.0 \\
3 & 4.71 & -93893 & 2.4 & N/A & 213.3 & 2100.0 \\
\hline
\end{tabular}

The universality of the relaxation time affords some significant advantages for certain applications of our model. For example, the estimations of the parameters $a, d$, and $f$ in Eq. (4) is done by linear least squares calculations, while those in Eq. (3) are estimated by nonlinear least squares calculations. This means that there would be no difficulty in obtaining global solutions of Eq. (4). Once the relaxation time has been estimated, users would not have to further solve nonlinear least square equations. 

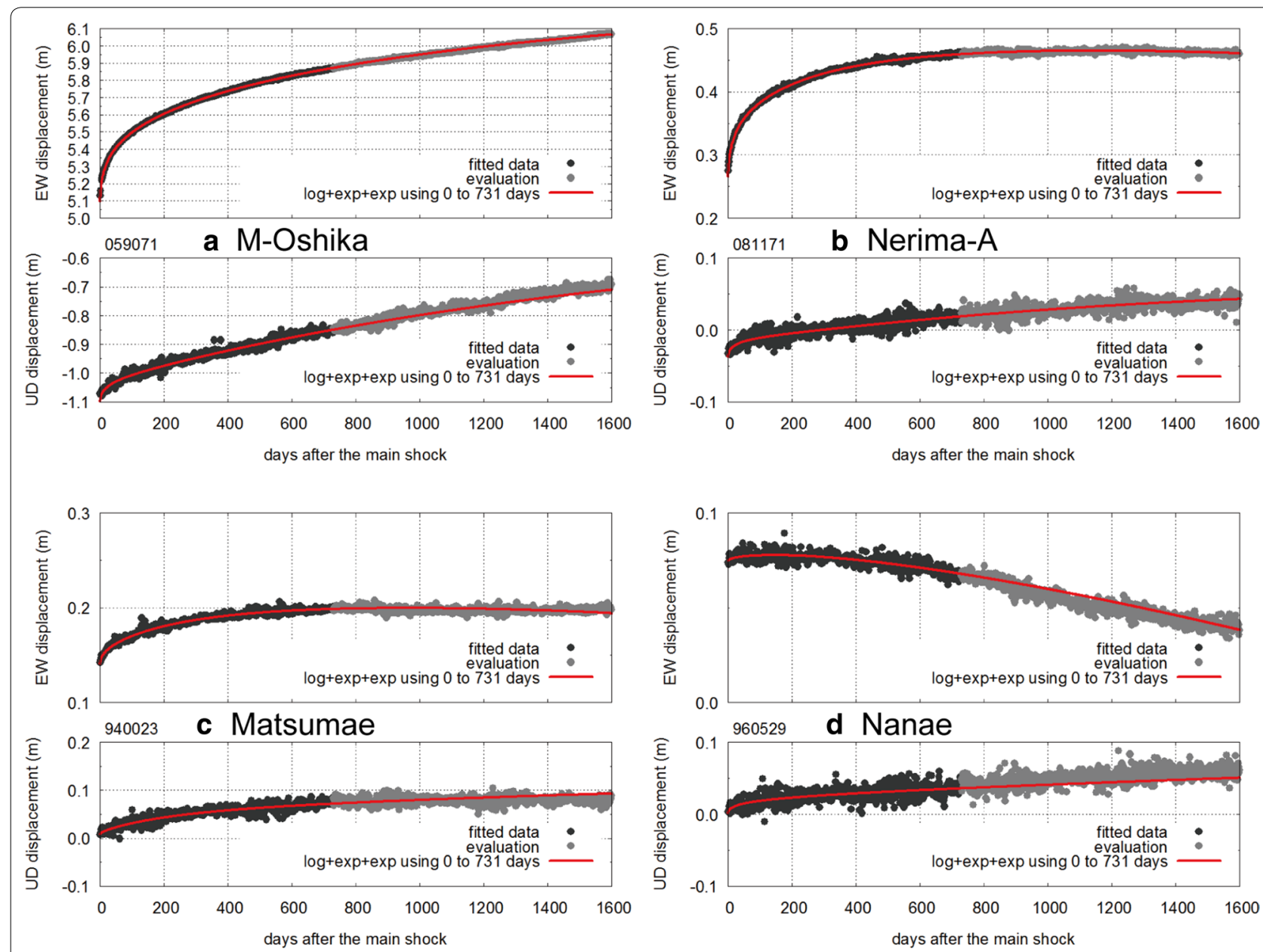

Fig. 6 Results of a fitting test performed using Model 2 and the given relaxation time constants of the GNSS time series at the four stations. The test was performed to examine the universality of the time constants estimated at the other stations. The notations are the same as in Fig. 3

One of such possible applications is the simple modelling of nonlinear motions of the International Terrestrial Reference Frame (ITRF) (IAG 2015), which requires a shortterm prediction performance.

The relaxation time constants presented in Table 1 are mainly for the evaluation of the prediction performance. Fortunately, we have the latest and more accurate estimates of the time constants compared to those listed in Table 1. For practical use, we recommend the following three equations with the latest relaxation time constants:

$$
\begin{aligned}
D(t)= & a \ln (1+t / 10.3)+c-d \exp \\
& (-t / 3306.0)+V t \\
D(t)= & a \ln (1+t / 1.47)+c+d \ln (1+t / 123.3) \\
& -f \exp (-t / 450021)+V t, \\
D(t)= & a \ln (1+t / 2.0)+c-d \exp (-t / 338.0) \\
& -f \exp (-t / 699997)+V t .
\end{aligned}
$$

The relaxation time constants of each of the above equations were estimated by nonlinear least squares calculations using the latest data obtained between 12 March 2011 and 17 October 2015. Because of the possible universal application of these equations over a wide area in Japan, they may be used to fit the GNSS time series of many stations in eastern Japan. The use of these equations to perform linear least squares calculations would enable easier calculation of postseismic displacements. Users can test each model and choose the one most suitable for their purpose.

In conventional approaches, there are cases in which the three components (east, north, and up) of a displacement have very different relaxation times, or different stations have very different relaxations times, or the estimated relaxation times are highly dependent on the fitting period. The findings of the present study suggest that such dependencies imply inappropriate selection of the fitting functions. Specifically, we found that 
a single set of relaxation times could be used to better explain the postseismic time series of the three components of all the considered stations. However, although there was an improvement in the stability of the relaxation times of our model with respect to the fitting period, the relaxation times still depended on the fitting period. This was probably because the three relaxation time constants still represented multiple numbers, or a broad spectrum, of relaxation times. This is one of the limitations of our model. Moreover, there may be better fitting functions than the functions proposed in this work. These issues would be addressed in a future work of ours.

As an application of our model, we considered the elimination of noise, namely the postseismic deformation of the Tohoku-Oki earthquake in the Tokai region. In a related study, by analysis of the velocity change of the GPS time series, Ozawa et al. (2002) detected a longterm slow-slip event (L-SSE) in the Tokai region between 2000 and 2005 at the boundary between the Eurasian and Philippine Sea plates. Although a similar velocity change on a smaller scale has been observed since 2013, the $\mathrm{S} / \mathrm{N}$ has been low mainly because of postseismic noise. Thus, the relationship between the small velocity change and the L-SSE was uncertain. The elimination of the noise has thus been an urgent need. The GSI applied Model 1 to their analysis of the recent velocity change of the GPS time series in the Tokai region and successfully eliminated the noise due to postseismic deformation, after which the L-SSE was detected (GSI 2015b). Although Model 1 has the poorest fitting and prediction performance among the three models presented in this paper, its simplicity affords two advantages under these conditions. Firstly, it enables modelling using low-S/N data observed far from the source region of the TohokuOki earthquake, such as in the Tokai region. Secondly, it could be used for a shorter fitting period in the early postseismic stage because the global solutions can be more easily obtained compared to when the other two models used.

\section{Relationship between postseismic deformation mechanisms and decay functions}

The determination of the relative contributions of the afterslip and viscoelastic relaxations to the postseismic deformation of the 2011 Tohoku-Oki earthquake was the primary objective of this study. Although this is very difficult to achieve, we attempted to determine the possible relationships between the respective mechanisms of the afterslip and viscoelastic relaxations and the exp and log decay functions of our model. Scholz (1990), Marone et al. (1991), and Scholz (1998) all proposed that the afterslip increases logarithmically with time. Freed et al.
(2007) and Gahalaut et al. (2008) also used a logarithmic function to fit GPS displacement time series. Furthermore, the postseismic surface deformations determined by Maxwell viscoelastic rheologies exhibited an exponential-like relaxation phase (Hetland and Hager 2006). Moreover, postseismic displacements calculated by models with biviscous Burgers rheologies are often described by logarithmic functions (Hetland and Hager 2006). Hoechner et al. (2011) also proposed that the response of a Burgers body consists of two superposed exponential decays.

Thus, the exponential term may represent the viscoelastic relaxation, while the logarithmic term probably includes the contribution of the afterslip and possibly that of the viscoelastic relaxation. Our results therefore may indicate that the contribution of the viscoelastic relaxation has been on an increase, while that of the afterslip has rapidly decreased. The superiority of Models 2 and 3 over Model 1 suggests a contribution of short-term viscoelastic relaxation, corresponding to the second term in Eqs. (2) and (3). This is consistent with the findings of Sun et al. (2014).

Hu et al. (2014) investigated the contributions of poroelastic rebound to the postseismic deformation of the Tohoku-Oki earthquake. Their test results indicate that the poroelastic contribution to the surface deformation is limited to the vicinity of the rupture area. Their model predicts $0-5 \mathrm{~cm}$ uplifts and horizontal displacements less than $2 \mathrm{~cm}$ on land. These minor contributions of poroelastic rebound are probably included in the GNSS time series as a minor factor.

\section{Function fitting of sum of multiple decay functions}

Our results show that the logarithmic decay function tends to be more suitable than the exponential decay function for fitting the postseismic deformation time series of the 2011 Tohoku earthquake. We investigated the possible reason for this by conducting simulation tests. We attempted to fit a single log or exp function to the sum of multiple decay functions with multiple timescale relaxations. We found that the sum of two log functions could always be better fitted by a $\log$ function than by an exp function. The sum of two or more exp functions with similar amplitudes and different relaxations time were also usually better fitted by a log function than by an exp function. Figure 7 shows two samples results of the simulation tests. In cases (a) and (b), the fitting performance of the log function (orange curve) was better than that of the exp function (blue curve). The superiority of the log function increased with increasing number of component functions of the fitting target. The overall results of the tests indicated that a single log function could be used to fit the sum of multiple decay functions 


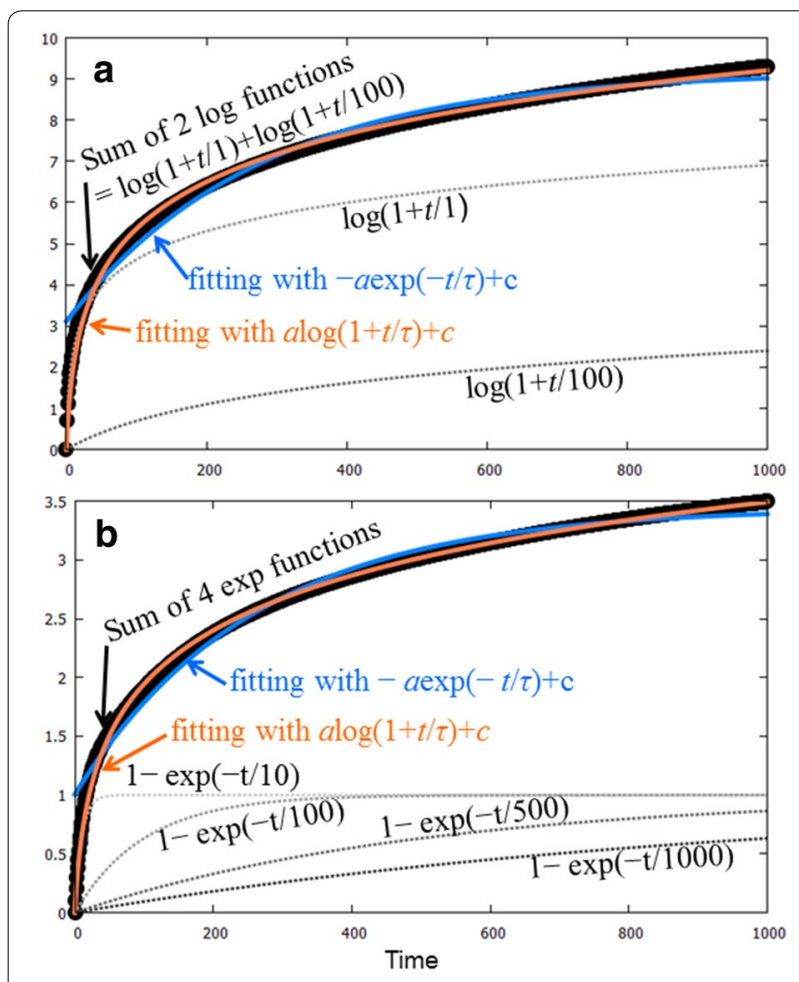

Fig. 7 Sample results of simulation tests on fitting the sum (black closed circles) of multiple decay functions (grey dotted lines) using a logarithmic function (orange curve) and an exponential function (blue curve), respectively

and could also be applied to a broad spectrum of relaxation times. Other similar simulation tests revealed that the sum of two exponential functions could be perfectly fitted by two exponential functions with a global solution. Even in this case, a single logarithmic function performed better than a single exponential function. It is, however, important to note that exp + exp data are more reasonably fitted using an exp + exp model than by using a single log model. Actually, an exp + exp model is most suitable for this case.

The degree of complexity of the fitting function may depend on the magnitude of the earthquake because of the following two reasons. Firstly, the decay time of the subsurface viscoelastic relaxation depends on the subsurface local conditions such as the viscosity and thickness of the material. Secondly, a larger earthquake generally has a wider source area with various subsurface local conditions. Thus, the postseismic surface deformation (produced by a combination of effects resulting from multiple subsurface deformation mechanisms) of a larger earthquake may have a broader spectrum of timescales, and more decay function terms with different timescales may therefore be required to fit the time series of the postseismic deformation. In this case, for the same number of relaxation time constants, a logarithmic function will be more advantageous for the fitting and prediction of the postseismic deformation compared to an exponential function.

\section{Limitations of combined log and exp models}

We assumed that the velocity $V$ in Eqs. (1)-(3) does not change, and we therefore used the average velocity between 1 April 1997 and 31 March 2000 as the steady velocity at each station. The reason why we did this rather than estimate $V$ was because the evolution of the exponential decay term (indicated by the light blue curves in Figs. 4,5 ) over a long relaxation time is similar to that of the velocity term (indicated by the green curves in Figs. 4, 5), and the correlation between the two is very high. This makes it very difficult to obtain a stable global solution. The period just before the Tohoku-Oki earthquake was not selected because Suito et al. (2011) suggested that large transient seaward displacements were observed at Iwate, Miyagi, Fukushima, and Ibaraki several years prior to the Tohoku-Oki earthquake, and we could therefore not obtain steady velocities for this period.

As shown in Figs. 3, 4, and 5, the future velocities will approach the assigned steady velocities. The long-term predictions of our models are thus highly dependent on the steady velocities and the long-term relaxation time constants. The assigned steady velocity is thus very important, especially for predictions. Future observations will either validate or invalidate the assumptions and predictions of the present work. In this context, our models may be the key to resolving the question of whether $V$ is constant throughout a seismic cycle.

To investigate this question, we conducted a test simulation to attempt an estimation of the steady velocity component. Actually, no reasonable stable global solution could be obtained by this procedure. Nevertheless, it was imperative for us to report the tendency of the estimated steady velocity for reference. Firstly, the estimated east component of the steady velocity of each of the four stations was negative (i.e. westward). Secondly, the estimated vertical component of the steady velocity in each of the stations was positive (indicating uplift), while the estimated long-term exp component was negative (indicating subsidence).

As we mentioned above, although the long-term prediction is highly dependent on the steady velocity $V$, it could not be estimated. This is the main prediction limitation of $\log$ and exp models. Other limitations constitute factors that may cause the actual future time series of the postseismic deformations to deviate from our predictions. They are as follows: (1) the limitation of the function fitting, (2) status change of the postseismic deformation mechanisms, (3) variable (inconstant) 
interseismic velocity, (4) future coseismic deformations, and (5) observation errors. The actual postseismic deformations could deviate from the predictions due to only some of the above factors. Hence, rather than to make predictions, the primary objective of this study was to develop new models for predicting postseismic deformations, demonstrate the capability of the proposed models, and provide fundamental information for better understanding of crustal activities.

\section{Conclusions}

1. We proposed combined logarithmic and exponential function models for fitting high-S/N GNSS postseismic deformation data of the 2011 Tohoku-Oki earthquake.

2. By simultaneous estimation of the common relaxation time constants of three-component GNSS data obtained from four stations with different vertical evolutions, we presented an improved method for obtaining global nonlinear least squares solutions.

3. The $\log +\log +\exp$ model (Model 2; Fig. 4) and $\log +\exp +\exp$ model (Model 3; Fig. 5) have significantly better fitting and short-term prediction performances than the conventional decay function models (e.g. Fig. 2).

4. Our findings suggest that multiple postseismic deformation mechanisms with different timescales have been active in eastern Japan.

5. Our results may indicate that the effect of viscoelastic relaxation on land GNSS stations has been on a gradual increase, while that of afterslip has rapidly decreased.

6. Limitations and potential: The predictions of our model were significantly dependent on the assigned velocities. Future observations are expected to determine the validity of the assigned values and predictions.

7. The proposed models, which were developed using relaxation time constants that were estimated only once, appear to be universally applicable, and this means that linear rather than nonlinear least squares calculations can be used for various applications.

8. As part of its monthly assessments, the Earthquake Assessment Committee in Japan used our model to detect the Tokai L-SSE, which has started in 2013.

\section{Acknowledgements}

We would like to thank Reiko Kai for preparing the GEONET data, Hisashi Suito for his valuable discussions, and Teruyuki Kato for his useful advice on the application of our model.

\section{Competing interests}

The author declares that he has no competing interests.
Received: 22 December 2015 Accepted: 3 March 2016

Published online: 12 March 2016

\section{References}

Akaike H (1974) A new look at the statistical model identification. IEEE Trans Autom Control 19:716-723

Freed AM, Bürgmann R, Herring T (2007) Far-reaching transient motions after Mojave earthquakes require broad mantle flow beneath a strong crust. Geophys Res Lett 34:L19302. doi:10.10/2007GL030959

Gahalaut VK, Jade S, Catherine JK, Gireesh R, Ananda MB, Kumar P, Narsaiah M, Jafri SSH, Ambikapathy A, Bansal A, Chadha RK, Gupta DC, Nagarajan B, Kumar S (2008) GPS measurements of postseismic deformation in the Andaman-Nicobar region following the giant 2004 Sumatra-Andaman earthquake. J Geophys Res 113:B08401. doi:10.1029/2007JB005511

Geospatial Information Authority of Japan (GSI) (2014) Crustal movements in the Tohoku district. Rep Coord Comm Earthq Predict 91:85-90 (in Japanese)

Geospatial Information Authority of Japan (GSI) (2015a) Crustal movements in the Tohoku District. Rep Coord Comm Earthq Predict 93(3-2):68-83

Geospatial Information Authority of Japan (GSI) (2015b) Time-dependent inversion analysis of L-SSE in Tokai region. In: Paper presented at the monthly meeting of the Earthquake Assessment Committee for Areas under Intensified Measures against Earthquake Disaster (EAC), JMA, Tokyo, Japan, 28 Sept 2015

Hashimoto M, Choosakul N, Hashizume M, Takemoto S, Takiguchi H, Fukuda Y, Fujimori K (2006) Crustal deformations associated with the great Sumatra-Andaman earthquake deduced from continuous GPS observation. Earth Planets Space 58:127-139

Hetland EA, Hager BH (2006) The effects of rheological layering on postseismic deformation. Geophys J Int 166:277-292

Hoechner A, Sobolev SV, Einarsson I, Wang R (2011) Investigation on afterslip and steady state and transient rheology based on postseismic deformation and geoid change caused by the Sumatra 2004 earthquake. Geochem Geophys Geosyst 12:Q07010. doi:10.1029/2010GC003450

Hu Y, Bürgmann R, Freymueller JT, Banerjee P, Wang K (2014) Contributions of poroelastic rebound and a weak volcanic arc to the postseismic deformation of the 2011 Tohoku earthquake. Earth Planets Space 66:106. doi:10.1186/1880-5981-66-106

IAG (2015) Commission 1-reference frames. http://iag.dgfi.tum.de/fileadmin/IAG-docs/Travaux2015/01_Travaux_Template_Comm_1_tvd.pdf Accessed 30 Nov 2015

Marone CJ, Scholz CH, Bilham R (1991) On the mechanics of earthquake afterslip. J Geophys Res 96:8441-8452

Nakagawa H, Toyofuku T, Kotani K, Miyahara B, Iwashita C, Kawamoto S, Hatanaka Y, Munekane H, Ishimoto M, Yutsudo T, Ishikura N, Sugawara Y (2009) Development and validation of GEONET new analysis strategy (version 4). J Geogr Surv Inst 118:1-8 (in Japanese)

Nishimura T (2014) Pre-, co-, and post-seismic deformation of the 2011 Tohoku-Oki earthquake and its implication to a paradox in short-term and long-term deformation. J Disaster Res 9:294-302

Ozawa S, Murakami M, Kaidzu M, Tada T, Sagiya T, Hatanaka Y, Yarai H, Nishimura T (2002) Detection and monitoring of ongoing aseismic slip in the Tokai region, central Japan. Science 298:1009-1012

Ozawa S, Nishimura T, Munekane H, Suito H, Kobayashi T, Tobita M, Imakiire T (2012) Preceding, coseismic, and postseismic slips of the 2011 Tohoku earthquake, Japan. J Geophys Res. doi:10.1029/2011JB009120

Scholz CH (1990) The mechanics of earthquake and faulting. Cambridge University Press, New York

Scholz CH (1998) Earthquakes and friction laws. Nature 391:37-42

Suito H, Nishimura T, Tobita M, Imakiire T, Ozawa S (2011) Interplate fault slip along the Japan Trench before the occurrence of the 2011 off the Pacific coast of Tohoku Earthquake as inferred from GPS data. Earth Planets Space 63:615-619. doi:10.5047/eps.2011.06.053

Sun T, Wang K (2015) Viscoelastic relaxation following subduction earthquakes and its effects on afterslip determination. J Geophys Res 120:1329-1344. doi:10.1002/2014JB011707 
Sun T, Wang K, linuma T, Hino R, He J, Fujimoto H, Kido M, Osada Y, Miura S, Ohta Y, Hu Y (2014) Prevalence of viscoelastic relaxation after the 2011 Tohoku-oki earthquake. Nature 514:84-87. doi:10.1038/nature13778 Takahashi H, Nakao S, Okazaki N, Koyama J, Sagiya T, Ito T, Ohya F, Sato K, Fujita Y, Hashimoto M, Hoso Y, Kato T, linuma T, Fukuda J, Matsushima T, Kohno Y, Kasahara M (2004) GPS observation of the first month of postseismic crustal deformation associated with the 2003 Tokachi-oki earthquake ( $\mathrm{M}_{\mathrm{JMA}}$ 8.0), off southeastern Hokkaido, Japan. Earth Planets Space 56:377-382

Tobita M, Akashi T (2015) Evaluation of forecast performance of postseismic displacements. Rep Coord Comm Earthq Predict 93:393-396 (in Japanese)

\section{Submit your manuscript to a SpringerOpen ${ }^{\circ}$ journal and benefit from:}

- Convenient online submission

- Rigorous peer review

- Immediate publication on acceptance

- Open access: articles freely available online

- High visibility within the field

- Retaining the copyright to your article

Submit your next manuscript at $\downarrow$ springeropen.com 